\title{
Psychological Aspects of Rehabilitation as Perceived by Physical Therapists
}

\author{
Alexa Knuth ${ }^{1}$, Lindsay Ross Stewart ${ }^{1 *}$, Corinne Brent ${ }^{1}$ and Ryan Salerno ${ }^{2}$ \\ ${ }^{1}$ Department of Applied Health, Southern Illinois University Edwardsville, USA \\ ${ }^{2}$ Department of Athletic Training, Southern Illinois University Edwardsville, USA
}

Submission: February 15, 2018; Published: February 26, 2018

*Corresponding author: Lindsay Ross Stewart, Department of Applied Health, Southern Illinois University Edwardsville, USA; Email: Irossst@siue.edu

\begin{abstract}
This study aimed to identify behaviors of patients that cope successfully and unsuccessfully with physical therapy, psychosocial strategies physical therapists currently use, psychosocial strategies physical therapists would like to become more educated on, and current counseling referral systems. The Physiotherapist and Sport Psychology Questionnaire was sent to all 50 physical therapy state associations with a request that they make it available to their members. Four organizations agreed to distribute the survey and 95 (77 females, 18 males) physical therapists participated in the study. Stress/anxiety, depression, and pain management problems were the most commonly perceived psychological conditions presented by patients. Exercise variety, short-term goal setting, and encouraging positive self-thoughts were used most often in assisting patients during their recovery. Physical therapists indicated they were most interested in learning more about how to use effective communication, understanding individual motivation, and enhancing self-confidence. Lastly, 65 physical therapists had referred their patients to counseling services although only eight had identified having a standard procedure for counseling referrals. These results indicate that physical therapists recognize the importance of the psychological component of rehabilitation and as a result apply psychosocial strategies during patient recovery. However, few are doing it systematically partially due to a lack of formal education and training as indicated by the results of this study.

Keywords: Psychology of Injury; Physical Therapy
\end{abstract}

Abbreviations: WHO: World Health Organization; ICF: International Classification of Function, Disability and Health; IRB: Institutional Review Board; PSPQ: Physiotherapist and Sport Psychology Questionnaire; CAPTE: Commission on Accreditation in Physical Therapy Education's

\section{Introduction}

An individual's injury treatment is influenced by emotional, behavioral, and situational factors [1,2]. Psychosocial factors, in relation to patients' experience of pain, may explain why some patients are unable to make a full recovery. The World Health Organization's (WHO), International Classification of Function, Disability and Health (ICF) recognizes pain as a subjective experience and have suggested healthcare professionals shift from treating in a biomedical perspective to a biopsychosocial perspective [3]. The biopsychosocial model recognizes the biological factors pertaining to the disease, injury, or condition, as well as the psychological factors (i.e., thoughts, feelings, and behaviors) and social factors (e.g., socio-demographics and social support) that contribute to the patient's pain and rehabilitation experience that could not otherwise be explained by the biomedical model [4].

Although the biopsychosocial model is considered a better model of explaining disease and disability by some, physical therapists report uncertainty about identifying and assessing psychosocial aspects within their practice [5]. Existing research has reported several psychological constructs as obstacles to recovery, including; pain perceptions [6-8], catastrophizing $[9,10]$, fear, anxiety, depression [11-15], anger [16-22], selfefficacy [23-26], sociodemographics [27-29] and a lack of social support [30,31]. Although assessment and management of all these obstacles is not suited for routine use, they do help to increase the understanding of how psychosocial factors impact the rehabilitation process.

Physical therapists' are considered to be in an optimal position to treat the psychosocial and physical factors accompanying the rehabilitation process [32-34]. However, many challenges with the integration of the biopsychosocial model during physical therapy have been reported [35]. First, physical therapists feel the need for additional training to better understand and treat the psychosocial component considering their primary education seldom covers these factors. Second, current screening tools for psychosocial factors are long and complicated to score, therefore making them impractical for everyday use. Third, not all psychosocial issues can be appropriately addressed during physical therapy and require outside specialists. Few physical therapists work in a setting where mental health specialists 
are accessible, which may further discourage therapists to assess psychosocial factors. Lastly, physical therapists are not reimbursed for psychosocial interventions further decreasing their likelihood of performing these assessments [36].

Current research investigating physical therapists' perceptions of the psychosocial content in their field is warranted [36]. Theoretical rationales referenced from similar fields are necessary to improve practitioners' clinical decisionmaking [1]. Although occupational therapy and chiropractic are often regarded as similar fields to physical therapy, research regarding psychosocial assessment within these fields is also limited $[37,38]$. Sports medicine has comprehensively examined the relationship between thoughts, feelings, and behaviors in regards to the psychological and behavioral responses of injury and recovery. Therefore, it currently serves as the best guide for psychosocial assessment and intervention within physical therapy.

An extensive amount of research in the field of sports medicine has investigated the psychosocial impact of athletic injuries [3941]. It is suggested that medical professionals, such as physical therapists, should provide psychological support during athlete rehabilitation, be able to effectively implement psychosocial interventions, and have the ability to recognize when referral is appropriate. Moreover, it has also been suggested that physical therapists who care for the psychological needs of their athletes improve patient-therapist relationships and communication with their patient. Good rapport and communication have been associated with greater adherence to the rehabilitation program, increased motivation, and better recovery.

The integrated model of interest was proposed to explain the psychological response to sport injury and the rehabilitation process. The model suggests a bi-directional, cyclic relationship between the athlete's cognitive appraisal, emotional response, and behavioral response to their injury and the rehabilitation process. Although this model is recognized as the most comprehensive model of the psychological response to sport injury [42], it does not account for the biological factors influencing recovery outcomes and thus cannot be the only model referenced by physical therapists.

In accordance, the biopsychosocial model of sport injury rehabilitation [43] was developed to explain the relationship between psychological factors and physical recovery. This model proposes possible explanations of how psychological factors impede the rehabilitation process. Unlike the integrated model, however, it does not explain the intricate relationship between different psychological factors also affecting the rehabilitation process. When used in conjunction, the integrated model of psychological response and the biopsychosocial model of sport injury rehabilitation provide a broader framework of the psychological and social factors impeding the rehabilitation program than the one originally outlined by Engel [4]. These combined models may better explain the complex relationship between biological, psychological, and social factors on rehabilitation and recovery outcomes, and could therefore serve as a better reference for clinical reasoning and decision-making compared to the biomedical model alone.

Until recently, limited research explored sport medicine professionals' perceptions of psychosocial content in their field [44]. Results from these studies strongly suggest that medical professionals find the psychological content in their field important and impactful to the rehabilitation process. While physical therapists reported that all athletes were psychologically affected by their injury, it is further suggested that physical therapists do not receive the appropriate training required to recognize and intervene psychological techniques. These studies provide the most comprehensive review of the psychosocial content of rehabilitation and medical professionals' current use of psychological interventions. However, the only population assessed in these studies was athletes. No research currently exists that evaluates the psychological content in other areas of physical therapy. Furthermore, a majority of these studies were done in the United Kingdom and may not be directly applicable to physical therapists working in the United States due to differences in education.

It is evident that physical therapists need to address both physical and psychological aspects of their patients. However, research outside of sports medicine on the psychological content within physical therapy is minimal. Furthermore, it has been argued that physical therapists are poorly prepared to treat patients under the biopsychosocial model, nor have they received the appropriate training necessary to implement psychological interventions. In order to help focus on meaningful and effective interventions, a greater account of patient behaviors and current psychosocial management styles should be evaluated. The purpose of this research is to extend the work of sports medicine psychology and investigate the views of physical therapists in the United States on the psychological responses and coping behaviors of their patients and the psychosocial strategies currently being used in the field of sport psychology.

\section{Methods}

\section{Participants}

All 50 state chapters of the American Physical Therapy Association were contacted via email. Chapters were asked to make the questionnaire (described below) available to their members by either email or a post on their website. One hundred and thirty-five physical therapists from Illinois, Georgia, New York, Arizona, California, Montana, Missouri, and Minnesota started the online questionnaire. Forty participants dropped out or only partially completed the questionnaires. Thus, 95 physical therapists (70\% response completion rate) completed the questionnaire. Participants consisted of 18 males (18.9\%; mean age $=36.94 \pm 10.48$ years) and 77 females $(81.1 \%$; mean age $=41.70 \pm 12.01$ years). A majority of the sample worked in an outpatient clinic or home health, respectively. The majority of 
participants had a Doctoral degree $(n=53 ; 55.8 \%)$ or a Master's degree $(n=18 ; 18.9 \%)$ as their highest education level.

\section{Measures}

Background Questionnaire: General background information about the physical therapists' demographics (i.e., sex, age, and race) and information regarding the therapists' occupations and education were collected, such as licensure, highest degree obtained, years in the practice, and current work setting. Included in this portion of the questionnaire were questions regarding sport psychologist and counseling availability to practitioners and, if any, past sport psychology education.

Physiotherapist and Sport Psychology Questionnaire (PSPQ) [34]: The PSPQ is an adapted version of the Athletic Trainer and Sport Psychology Questionnaire [45]. The PSPQ (Appendix A) is a self-report questionnaire exploring physical therapists' perspectives on the psychological content of their practice. The questionnaire contains 11 items of both a quantitative (9) and qualitative (2) nature. The PSPQ is divided into 4 sections: the perceived psychological response and implications of musculoskeletal conditions, coping behaviors of successful and unsuccessful recovery, psychosocial strategies therapists are currently using and strategies therapists need more education on, and current referral systems to counseling.

Question 1 asks the physical therapist to rate how often they encounter 9 different psychological responses found to be associated with injuries on a 1-5 Likert-type scale from never to very often. Questions 2 and 3 are open-ended questions asking the participant to list the top 4 behaviors of patients whom cope successfully and unsuccessfully with their condition. Questions 4,5 , and 6 regard patient referral systems to counseling services, and are in a 'Yes' or 'No' format.

Question 7 is a list of 13 psychological interventions, such as goal-setting and encouraging positive self-talk, that asks therapists to rate how often they have used each intervention. All items are measured on a 1-5 Likert-type scale from never to $100 \%$ of the time. Question 8 asks the participant to rate how often their patient is psychologically affected by their treatment measured on a $1-5$ Likert-type scale ( $1=$ never, $5=100 \%$ of the time). On question 9, the participant is asked to rate on a 1-5 Likert-type scale (1=not important, 5 =very important) 14 psychological interventions they feel would be beneficial to learn more about. Question 10 asks the participant to rate how important they feel it is to treat the psychological aspect of their field. Question 11 rates the extent the therapist feels prepared to treat the psychological aspect. Therapists rated these final two questions on a 1-4 Likert-type scale from not prepared/ important to very prepared/important.

A similar version of the PSPQ was used in preliminary studies by Hemmings [34] and Arvinen Barrow [40]. No psychometric proprieties were reported for in either questionnaire. For the purposes of this study, 'physiotherapist' was changed to 'physical therapist' in accordance with the American professional title and 'athletic injuries' was changed to 'musculoskeletal disorder(s)/ diseases(s)' to encompass the variety of conditions physical therapists treat.

\section{Procedure}

Following Institutional Review Board (IRB) approval, email addresses of all 50 chapters of the APTA were obtained from their website. All chapters' presidents or board members were sent an email requesting they make the questionnaire available to their members. The email provided an introduction of the research and a link to complete the questionnaire on Qualities. Participants simply clicked the link provided to them by their chapter (via email or a statement on the chapter webpage). The online link included an informed consent form, demographics, and the PSPQ. Participants were not required to provide their name or any other identifying information. After collection, data was analyzed through SPSS software.

\section{Results}

\section{Psychological Impact of Musculoskeletal Conditions}

Descriptive statistical analyses showed that nearly all physical therapists $(n=94 ; 98.9 \%)$ believed that patients were psychologically affected by their condition to some degree $(m=3.83, \mathrm{sd}=1.09)$. In addition, of the nine listed psychological conditions patients could face, the participants rated stress or anxiety $(m=4.39, \mathrm{sd}=0.72)$, depression $(\mathrm{m}=4.07$, $\mathrm{sd}=0.84)$, and problems with pain management $(\mathrm{m}=4.00, \mathrm{sd}=0.81)$ as the most commonly encountered conditions (Table 1). Overall, $89 \%$ of the participants believed that addressing these aspects was either important or very important.

Table1: Psychological Conditions Encountered by Physical Therapists.

\begin{tabular}{|c|c|c|}
\hline Psychological Response & Mean & SD \\
\hline Stress/Anxiety & 4.39 & 0.72 \\
\hline Depression & 4.07 & 0.84 \\
\hline Problems with Pain Management & 4.00 & 0.81 \\
\hline Problems with Treatment Adherence & 3.69 & 0.91 \\
\hline Problems with Motivation & 3.67 & 0.92 \\
\hline Problems with Confidence & 3.39 & 0.92 \\
\hline Problems with Attention or Concentration & 3.08 & 1.07 \\
\hline Anger & 3.02 & 0.96 \\
\hline Exercise Addiction & 2.28 & 0.92 \\
\hline
\end{tabular}

Note: $1=$ Never encounter; $5=$ Very often encounter

\section{Patients' Coping Characteristics}

Participants were asked to identify the top 4 characteristics in patients that cope successfully and unsuccessfully with the rehabilitation and treatment program. A thematic analysis was used to categorize data. Categories created in Arvinen Barrow [40] and Clement et al. [33] were used as a guideline for the current study. 
Successful Coping Characteristics: Physical therapists revealed several different coping behaviors in patients that cope successfully with rehabilitation treatment. In total, four main themes were coded from the participants' responses. The results showed that patients who are "compliant to the rehabilitation program" (n=38), have a "positive attitude" (n=35), are "motivated" (n=25), and have an "understanding of injury and rehabilitation" $(\mathrm{n}=22)$ tend to cope more successfully with their injuries.

7.2.2 Unsuccessful Coping Characteristics: Similarly, four themes of psychological behaviors were coded from responses. The most frequently reported characteristics of patients who cope less successfully with their injury were "poor compliance to rehabilitation program" ( $n=36)$, "stress/anxiety" ( $n=34)$, "low motivation to rehabilitation program" (n=29), and "depression" $(\mathrm{n}=28)$.

\section{Psychological Interventions Current Use and Further Training}

Table 2: Psychological Interventions Currently Used By Physical Therapists.

\begin{tabular}{|c|c|c|}
\hline Psychological Technique & Mean & SD \\
\hline Creating Variety in Rehab Exercises & 4.42 & 0.68 \\
\hline Using Short-Term Goals & 4.41 & 0.84 \\
\hline Encouraging Positive Self-Thoughts & 4.07 & 0.99 \\
\hline Encouraging Effective Communication Skills & 3.92 & 1.03 \\
\hline Reducing Stress and Anxiety & 3.67 & 1.02 \\
\hline Enhancing Self-Confidence & 3.58 & 1.06 \\
\hline Using Relaxation Techniques & 3.39 & 1.22 \\
\hline Teaching Muscular Relaxation Techniques & 3.23 & 1.07 \\
\hline Improving Social Support & 3.21 & 1.05 \\
\hline Helping Patient Maintain Social Support & 3.11 & 1.13 \\
\hline Other Techniques (not specified) & 3.00 & 1.41 \\
\hline Reducing Depression & 2.90 & 1.17 \\
\hline Teaching Emotional Control Strategies & 2.83 & 1.28 \\
\hline
\end{tabular}

Note: $1=$ Never use; $5=$ Use $100 \%$ of the time

Participants were asked to indicate the frequency with which they used 13 specific psychological interventions (Table 2). The top interventions currently used by therapists were "creating variety in rehab exercises" $(m=4.42, \mathrm{sd}=0.68)$, "using short-term goals" ( $\mathrm{m}=4.41, \mathrm{sd}=0.84)$, and "encouraging positive self-thoughts" $(\mathrm{m}=4.07, \mathrm{sd}=0.99)$. The interventions used the least were "reducing depression" $(\mathrm{m}=2.90, \mathrm{sd}=1.17)$ and "teaching emotional control strategies" $(\mathrm{m}=2.83, \mathrm{sd}=$ 1.28). Furthermore, participants were also asked to indicate which techniques they believed they should be more educated on. Physical therapists rated which interventions they deem important for further training (Table 3). Therapists expressed that further training in "understanding individual motivation" $(m=4.27, s d=0.87)$, "using effective communication" $(m=4.27$, $\mathrm{sd}=1.02)$, and "enhancing self-confidence" $(\mathrm{m}=4.20, \mathrm{sd}=1.02)$ would best improve their quality of care for patients. The lowest rated interventions were "creating variety in rehab exercises" $(\mathrm{m}=3.77, \mathrm{sd}=1.26)$ and "teaching the use of mental imagery" $(\mathrm{m}=3.71, \mathrm{sd}=1.11)$.

Table 3: Psychological Interventions Physical Therapists Want Further Training In.

\begin{tabular}{|c|c|c|}
\hline Psychological Technique & Mean & SD \\
\hline Understanding Individual Motivation & 4.27 & 0.87 \\
\hline Using Effective Communication & 4.27 & 1.02 \\
\hline Enhancing Self-Confidence & 4.20 & 1.02 \\
\hline Reducing Stress and Anxiety & 4.19 & 0.92 \\
\hline Encouraging Positive Self-Thoughts & 4.17 & 0.96 \\
\hline Setting Realistic Goals & 4.12 & 1.13 \\
\hline Enhancing Listening Skills of Therapist & 4.10 & 1.15 \\
\hline Reducing Depression & 4.00 & 1.05 \\
\hline Teaching Emotional Control Strategies & 3.95 & 1.00 \\
\hline Improving Social Support & 3.83 & 1.13 \\
\hline Teaching Muscular Relaxation Techniques & 3.82 & 1.08 \\
\hline Teaching Concentration Skills & 3.81 & 1.04 \\
\hline Creating Variety in Rehab Exercises & 3.77 & 1.26 \\
\hline Teaching the Use of Mental Imagery & 3.71 & 1.11 \\
\hline
\end{tabular}

Note: $1=$ Not important; $5=$ Very important

\section{Current Referral Systems to Counseling}

Lastly, participants were asked about access and referral procedures to counseling. A total of 65 physical therapists (68\%) reported referring a patient for counseling services regarding their musculoskeletal condition. Responses revealed that only eight $(0.08 \%)$ of participants had a standard written procedure for referring patients to counseling services.

\section{Discussion}

\section{Psychological Impact of Musculoskeletal Conditions}

The purpose of this study was to investigate the views of licensed physical therapists working in the United States regarding the psychological content in their field as well as to obtain a greater account of patient behaviors observed in rehabilitation. Nearly all physical therapists (98.9\%) surveyed reported their patients to be psychologically affected by their musculoskeletal condition. Due to the high number of physical therapists reporting psychological effects due to musculoskeletal conditions, it is reasoned that psychological coursework should be formally implemented into physical therapist education. Physical therapists also specified that patients experienced a variety of different psychological responses in rehabilitation. The top rated psychological responses observed within physical therapy are 'stress/anxiety', 'depression', and 'problems with pain management'. These results are parallel to the findings in past research. However, 'Exercise Addiction' was reported as the 
second most observed psychological response in sport injury, whereas findings from the present study revealed it to be the least observed psychological response.

This discrepancy is not surprising as exercise addiction is considered a unique emotional response to an injured athlete compared to a non-athlete in physical therapy. Specifically, exercise addiction is the development of physical and psychological withdrawal symptoms from a lack of physical activity, which can be related to a loss of self-identity. Given that an extensive amount of an athlete's self-identity is derived from athletic performance, injured athletes are an at-risk population for developing an exercise addiction. However, other populations would not be expected to elicit such a response considering their self-identity is not based on an athletic identity. Consistent with previous research, stress and anxiety were reported as the top rated psychological responses to injury. Stress and anxiety affect injury vulnerability and recovery outcomes. Understanding the relationship between cognitive appraisals and emotional and behavioral responses has practical implications for physical therapists. Existing literature suggests practitioners working in rehabilitation settings are in an optimal position to not only treat the physical component but also teach coping skills to meet challenges of recovery and daily life.

Although stress and anxiety are frequently reported, strategies to reduce stress and anxiety, such as relaxation techniques and mental imagery, were among the least used psychological techniques. It has previously been suggested that a lack of knowledge in the use of these strategies and their potential benefits as well as a lack of training in the implementation may cause therapists to use strategies with which they are more confident using. Nevertheless, stress and anxiety are consistently reported as a barrier to the rehabilitation process. This suggests that further training and education for physical therapists is necessary to effectively access and manage these behavioral responses.

\section{Patients' Coping Characteristics}

Physical therapists surveyed were asked to report behaviors in patients who cope successfully and unsuccessfully with treatment. "Compliance to the Rehabilitation Program", "Positive Attitude", and "Motivation" were the top characteristics of patients that cope successfully. Conversely, "Poor Compliance", "Stress/Anxiety", and "Low Motivation to Rehabilitation Program" were observed as the top characteristics of patients that cope unsuccessfully. Findings from the current study mirror those of previous studies with similar findings.

Treatment adherence and compliance were rated among the top behaviors in successfully coping in both physical therapy and athletic training. This suggests that patients who regularly attend their treatment sessions and follow the treatment program are more likely to make a full recovery than those who don't. This is supported by previous findings that demonstrate higher percentages of adherence to physical therapy guidelines result in better improvements of physical outcomes and fewer treatment sessions [46]. Furthermore, the above findings highlight patient behaviors that should be encouraged during rehabilitation. Additionally, these findings provide clarity on interventions that would be useful in minimizing behaviors of patients who are perceived as coping unsuccessfully [47-51]. Future research should investigate the use of psychological interventions on patient's ability to respond appropriately and successfully? Cope with treatment. Specifically, psychological interventions from sport medicine literature have focused on the use of goal setting, imagery, relaxation techniques, self-talk, and social support.

\section{Psychological Interventions Current Use and Further Training}

The current sample reported using a variety of different psychological techniques in their practice. The top rated psychological techniques were "creating variety in rehabilitation exercises", "using ahort-term goal-setting", and "encouraging positive self-thoughts". Therapists also reposted a desired to learn more about "understanding individual motivation", "understanding effective communication", and "enhancing selfconfidence". Again, these findings are similar to those of Arvinen Barrow [40] and Hemmings [34]. These findings also supports existing research that there is a growing interest in physical therapists' desire to integrate psychological interventions within their practice [52].

Given the above evidence, the incorporation of psychology into formal physical therapy education seems warranted. However, the observed rate of physical therapists that had previously taken a course in psychology was 13\%. Current physical therapy education seldom addresses psychosocial factors. The Commission on Accreditation in Physical Therapy Education's (CAPTE) accreditation handbook does not require physical therapy programs to include specific clinical psychology coursework. Nor does the Guide to Physical Therapist Practice make any recommendations on the assessment and management of psychosocial factors [53]. This lack of knowledge and training could explain why interventions, such as relaxation techniques and mental imagery that are well supported in research are not expressed as areas of interest in future training by physical therapists. Nonetheless, the acknowledgment, assessment, and treatment of psychological disruptions are important. Therefore, future research is needed on the best methods of implementing psychosocial education to physical therapists.

\section{Current Referral Systems to Counseling}

The observed rate of referral by physical therapists to a psychologist in the current study was $68 \%$. In contrast, Hemmings [34] identified a referral rate of $9 \%$ and Arvinen Barrow [40] reported a rate of $24 \%$. At this time, it is unclear why this disproportionate variance exists. Future research should investigate the differences in referral methods between these populations. 


\section{Limitations and Future Research}

While the PSPQ was useful in comparing findings of similar studies, there were a few limitations with this measure. First, it is possible that terminology used in the PSPQ was left to interpretation by the participant. No psychological responses were further defined within the survey, making it possible that the participant misinterpreted the meaning. Short-term goal setting was found to be a strategy used frequently by physical therapists. However, in order for goal setting to be effective, the administrator must follow the S.M.A.R.T.S guidelines. Without formal training on goal setting it is possible that physical therapists implement goal setting in a way that hinders its efficacy.

Furthermore, the PSPQ may not be directly applicable to physical therapists working with populations other than athletes. As demonstrated previously, certain psychological responses are unique to specific populations that may not apply to the general public. In addition, although the researchers attempted to gain a nationally representative sample, this study was not nationally representative. Future research using a larger sample size is needed to fully understand physical therapists' perceptions of the psychological aspects in patient recovery. Additionally, future research should investigate accurate assessments of psychosocial content that is suitable for routine use. More information is also necessary to determine whether or not psychological interventions (e.g., goal-setting, imagery, and relaxation techniques) referenced from sport medicine can be applied to other populations within physical therapy. Lastly, research should aim to acquire more in-depth knowledge from the patient's perspective on the role of physical therapists and meaningful psychosocial interventions $[54,55]$.

\section{Conclusion}

In summary, physical therapists view the psychological content of their field to be important in the recovery of their patients. Psychological factors substantially impact patients' physical therapy experience and the associated outcomes. However, they are greatly overlooked in both practice and in education. The physical therapists surveyed in this study explained a variety of psychosocial issues they encounter on a daily basis in a variety of settings. A majority of the psychological skills physical therapists are currently using are aimed towards the psychosocial issues seen most commonly in therapy. However, some therapists expressed that they did not feel confident in treating the psychosocial issues associated with rehabilitation, even though it is known these factors play a major role in the recovery of patients.

This study, along with past research, demonstrates the clear need for physical therapists to be educated on the psychological needs of their patients. Education is essential for preparing these practitioners. It is suggested that emphasis be placed on teaching therapists theories of psychological constructs of rehabilitation and the practical application of psychosocial strategies. Although there were found to be subtle differences in the results, this study supports previous research regarding the importance of the psychological content within physical therapy. Furthermore, this suggests that theoretical frameworks and psychological interventions from the field of sports medicine may serve as an adequate guide in the future for training and educating entry-level physical therapists.

\section{References}

1. Brunner E, De Herdt A, Minguet P, Baldew S, Probst M (2013) Can cognitive behavioral therapy based strategies be integrated into physiotherapy for the prevention of chronic low back pain? A systematic review. Disability and Rehabilitation 35(1): 1-10.

2. Taylor J, Taylor S (1997) Psychological approaches to sports injury rehabilitation. Aspen 15(3): 201-202.

3. World Health Organization (2001) The International Classification of Functioning, Disability and Health (ICF). WHO, Geneva, Europe.

4. Engel G (1977) The need for a new medical model: A challenge for biomedicine. Science 196(4286): 129-136.

5. Foster N, Delitto A (2011) Embedding psychosocial perspectives within clinical management of low back pain: Integration of psychosocially informed management principles into physical therapist practicechallenges and opportunities. Physical Therapy 91(5): 790-803.

6. Mc Cracken L, Vowles K, Eccleston C (2004) Acceptance of chronic pain: Component analysis and a revised assessment method. Pain 107(1-2): 159-166.

7. Osborne T, Jensen M, Ehde D, Hanley M, Kraft G (2007) Psychosocial factors associated with pain intensity, pain-related interference, and psychological functioning in persons with multiple sclerosis and pain. Pain 127(1-2): 52-62.

8. Williams D, Thorn B (1989) An empirical assessment of pain beliefs. Pain 36(3): 351-358.

9. Baird A, Sheffield D (2016) The relationship between pain beliefs and physical and mental health outcome measures in chronic low back pain: Direct and indirect effects. Healthcare 4(3): 58-69.

10. Quartana P, Campbell C, Edwards R (2009) Pain catastrophizing: A critical review. Expert Review Neurotherapeutics 9(5): 745-758.

11. Connolly F, Aitken L, Tower M (2013) An integrative review of selfefficacy and paint recovery post acute injury. Journal of Advanced Nursing 70(4): 714-728.

12. Keefe F, Wilkins R, Cook W, Crisson J, Muhlbaier L (1986) Depression, pain, and pain behavior. Journal of Consulting and Clinical Psychology 54(5): 665-669.

13. Leeuw M, Goossens M, Linton S, Crombez G, Boersma K, et al. (2007) The fear-avoidance model of musculoskeletal pain: Current state of scientific evidence. Journal of Behavioral Medicine 30(1): 77-94.

14. Vlaeyen J, Linton S (2000) Fear-avoidance and its consequences in chronic musculoskeletal pain: A state of the art. Pain 85(3): 317-332.

15. Williams L, Jacka F, Pasco J, Dodd S, Berk M (2006) Depression and pain: An overview. ACTA Neuropsychiatrica 18(2): 79-87.

16. Bruehl S, Burns J, Chung 0, Chont M (2009) Pain-related effects of trait anger expression: Neural substrates and the role of endogenous opioid mechanisms. Neuroscience and biobehavioral reviews 33(3): 475-491.

17. Bruehl S, Burns J, Chung O, Ward P, Johnson B (2002) Anger and pain sensitivity in chronic low back pain patients and pain-free controls: The role of endogenous opiods. Pain 99(1-2): 223-233.

18. Bruehl S, Chung O, Burns J (2006) Anger expression and pain: An overview of findings and possible mechanisms. Journal of Behavioral Medicine 29(6): 593-606. 
19. Kerns R, Rosenberg R, Jacob M (1994) Anger expression and chronic pain. Journal of Behavioral Medicine 17(1): 57-67.

20. Lombardo E, Tan G, Jensen M, Anderson K (2005) Anger management style and associations with self-efficacy and pain in male veterans. Journal of Pain 6(11): 765-770.

21. Nisenzon A, George S, Beneciuk J, Wandner L, Torres C, et al. (2014) The role of anger in psychosocial sub grouping for patients with low back pain. Journal of Pain 30: 501-509.

22. Spielberger C, Johnson E, Russell S, Crane R, Jacobs G, et al. (1985) The experience and expression of anger: Construction and validation of an anger expression scale. In MA Chesney, RH Rosenman (Eds.), Anger and hostility in cardiovascular and behavioral disorders. Hemisphere, McGraw-Hill, New York, USA, p. 5-30.

23. Bandura A (1997) Self-efficacy: The exercise of control. WH Freeman and Company, New York, USA.

24. Osborne T, Jensen M, Ehde D, Hanley M, Kraft G (2007) Psychosocial factors associated with pain intensity, pain-related interference, and psychological functioning in persons with multiple sclerosis and pain. Pain 127(1-2): 52-62.

25. Jackson T, Wang Y, Wang Y, Huiyong F (2014) Self-efficacy and chronic pain outcomes: A meta-analytic review. The Journal of Pain 15(8): 800814.

26. Soderlund A, Äsenlöf P (2010) The mediating role of self-efficacy expectations and fear of movement and (re)injury beliefs in two samples of acute pain. Disability and Rehabilitation 32(25): 21182126.

27. Brekke M, Hjortdahl P (2004) Musculo skeletal pain among 40- and 45-year olds in Oslo: Differences between two socioeconomically contrasting areas, and their possible explanations. International Journal for Equity in Health 3: 1-5.

28. Jackson T (1999) Differences in psychosocial experiences of employed, unemployed, and student samples of young adults. Journal of Psychology 133: 49-60.

29. Rollman G, Laustenbacher S (2001) Sex differences in musculoskeletal pain. Clinical Journal of Pain 17(1): 20-24.

30. Jenson M, Moore M, Bockow T, Ehde D, Engel J (2011) Psychosocial factors and adjustment to chronic pain in persons with physical disabilities: A systematic review. Archives of Physical Medicine and Rehabilitation 92(1): 146-160.

31. Newton John T (2002) Solicitousness and chronic pain: A critical review. Pain Reviews 9(1): 7-27.

32. Arvinen Barrow M, Penny G, Hemmings B, Corr S (2010) UK chartered physiotherapists' personal experiences in using psychological interventions with injured athletes: An interpretative phenomenological analysis. Psychology of Sport and Exercise 11(1) 58-66.

33. Clement D, Granquist M, Arvinen Barrow M (2013) Psychosocial aspects of athletic injuries as perceived by athletic trainers. Journal of Athletic Training 48(4): 512-521.

34. Hemmings B, Povey L (2002) Views of chartered physiotherapists on the psychological content of their practice: A preliminary study in the United Kingdom. British Journal of Sports Medicine 36(1): 61-64.

35. Sanders T, Foster N, Bishop A, Ong N (2013) Biopsychosocial care and the physiotherapy encounter: Physiotherapists' accounts of back pain consultations. BMC Musculoskeletal Disorders 14: 1-10.

36. Nielsen M, Keefe F, Bennell K, Jull G (2014) Physical therapist-delivered cognitive-behavioral therapy: A qualitative study of physical therapists' perceptions and experiences. Physical Therapy 94(2): 197-209.
37. AJOT (2004) Psychosocial Aspects of Occupational Therapy. American Journal of Occupational Therapy 58(6): 669-672.

38. Langworthy J, Breen A (2007) Psychosocial factors and their predictive value in chiropractic patients with low back pain: A prospective inception cohort study. Chiropractic \& Osteopathy 15: 1-7.

39. Andersen M, Williams J (1999) Athletic injury, psychosocial factors, and perceptual changes during stress. Journal of Sports Sciences 17(9): 735-741.

40. Arvinen Barrow M (2009) Psychological rehabilitation from sport injury: issues in training and development of chartered physiotherapists. PhD thesis. University of Northampton, England, UK.

41. Wiese Bjornstal D, Smith A, Shaffer S, Morrey M (1998) An integrated model of response to sport injury: Psychological and sociological dynamics. Journal of Applied Sport Psychology 10(1): 46-69.

42. Kolt G (2003) Psychology of injury and rehabilitation. In GS Kolt, L Snyder Mackler (Eds.), Physical therapies in sport and exercise, Churchill Livingstone, London, pp. 165-183.

43. Brewer B, Andersen M, Van Raalte J (2002) Psychological aspects of sport injury rehabilitation: Toward a biopsychological approach. In DI Mostofsky, LD Zaichkowsky (Eds.), Medical aspects of sport and exercise. Fitness Information Technology, West Virginia, USA, pp. 4154.

44. Larson G, Starkey C, Zaichkowsky L (1996) Psychological aspects of athletic injuries as perceived by athletic trainers. Sport Psychology 10(1): 37-47.

45. Heaney C (2006) Physiotherapists' perceptions of sport psychology intervention in professional soccer. International Journal of Sport and Exercise Psychology 4(1): 67-80.

46. Rutten G, Degen S, Hendriks E, Braspenning J, Harting J, et al. (2010) Adherence to clinical practice guidelines for low back pain in physical therapy: Do patients benefit? Physical therapy 90(8): 1111-1122.

47. AIHW (2014) Health-care expenditure on arthritis and other musculoskeletal conditions 2008-2009.

48. American Physical Therapy Association (2015) Who are physical therapists?

49. Bailey K, Carleton N, Vlaeyen J, Asmundson G (2010) Treatments addressing pain-related fear and anxiety in patients with chronic musculoskeletal pain: A preliminary review. Cognitive Behaviour Therapy 39(1): 46-63.

50. Carter S, Rizzo J (2007) Use of outpatient physical therapy services by people with musculoskeletal conditions. Journal of the American Physical Therapy Association 87(5): 497-512.

51. Daykin A, Richardson B (2014) Physiotherapists' pain beliefs and their influence on the management of patients with chronic low back pain. Spine 29(7): 783-795.

52. Johnstone R, Donaghy M, Martin D (2004) A pilot study of a cognitivebehavioural therapy approach to physiotherapy, for acute low back pain patients, who show signs of developing chronic pain. Advances in Physiotherapy 2: 182-188.

53. Patel R, Chu S, Gerstman B (2014) Prevalence of musculoskeletal pain in adults with developmental disabilities. International Journal of Physical Medicine Rehabilitation 5(1): 8.

54. Santi G, Pietrantoni L (2013) Psychology of sport injury rehabilitation: A review of models and interventions. Journal of Human Sport Exercise 8(4): 1029-1044.

55. Yelin E (2003) Cost of musculoskeletal diseases: Impact of work disability and functional decline. The Journal of Rheumatology 68 8-11. 
